\title{
Cluster reducibility of multiquark operators
}

\author{
Wolfgang Lucha, ${ }^{1, *}$ Dmitri Melikhov ${ }^{1,2,3, \dagger}$ and Hagop Sazdjian $\oplus^{4, \ddagger}$ \\ ${ }^{1}$ Institute for High Energy Physics, Austrian Academy of Sciences, \\ Nikolsdorfergasse 18, A-1050 Vienna, Austria \\ ${ }^{2}$ D. V. Skobeltsyn Institute of Nuclear Physics, M. V. Lomonosov Moscow State University, \\ 119991 Moscow, Russia \\ ${ }^{3}$ Faculty of Physics, University of Vienna, Boltzmanngasse 5, A-1090 Vienna, Austria \\ ${ }^{4}$ Institut de Physique Nucléaire, Université Paris-Sud, CNRS-IN2P3, \\ Université Paris-Saclay, 91405 Orsay, France
}

(Received 28 August 2019; published 15 November 2019; corrected 2 December 2019)

\begin{abstract}
It is shown that the multiquark gauge-invariant operators can, in general, be decomposed into combinations of products of ordinary hadronic operators, exhibiting their cluster reducibility. The latter property inhibits the formation of completely compact multiquark bound states. Multiquark operators still play a crucial role in the description of exotic states in regions of configuration space where the hadronic clusters are close to each other. Our proof gives a foundation for a unified viewpoint, where the multiquark-type and the molecular-type approaches play complementary roles, at the gauge-invariant nonlocal operator level.
\end{abstract}

DOI: 10.1103/PhysRevD.100.094017

\section{INTRODUCTION}

The possibility of the existence of multiquark states [1,2], i.e., of states containing more than a pair of valence quark-antiquark for mesons and more than three valence quarks for baryons, also called exotic states, raises in QCD the following question: can QCD produce, by the sole confining forces, multiquark bound states in the same way as it produces ordinary hadrons?

An indication that multiquark states are not of the completely confined type arises from the observation that interpolating local currents, that might couple to them, can always, by Fierz rearrangements, be brought into forms where they appear as combinations of products of colorsinglet quark bilinear and/or trilinear local operators $[2,3]$. As a consequence, correlation functions of such currents become dominated by disconnected diagrams, representing free hadron propagators, while the connected diagrams depict interactions among hadrons, which are of the nonconfining type.

It might seem that the latter property concerns only some particular aspects of multiquark states, since their couplings

\footnotetext{
*wolfgang.lucha@oeaw.ac.at

†dmitri_melikhov@gmx.de

sazdjian@ipno.in2p3.fr
}

Published by the American Physical Society under the terms of the Creative Commons Attribution 4.0 International license. Further distribution of this work must maintain attribution to the author(s) and the published article's title, journal citation, and DOI. Funded by SCOAP. to local currents involve only a few moments of their wave functions. A wider view of their structure is provided by the general gauge-invariant states constructed with the aid of path-ordered gluon-field phase factors, also called Wilson lines, which are the color parallel transporters of the theory. One thus naturally arrives at the "string-junction" or " $Y$-shaped-junction" representation of multiquark states $[4,5]$, also characterized as "compact" states. Here, one expects to exhibit more easily their confined nature by means of their bound-state spectrum, which should show up through a tower of states lying at non-negligible distances from the multiquark thresholds, in analogy with the ordinary-hadron cases. A realization of the $Y$-shapedtype interactions is provided by the diquark picture [6-8].

The purpose of the present article is to show that even the general gauge-invariant multiquark operators are cluster reducible, in the sense that they are decomposable into a combination of products of gauge-invariant bilinear or trilinear operators, reminiscent of ordinary hadronic operators, thus generalizing the phenomenon encountered with local interpolating currents. ${ }^{1}$

The cluster reducibility property of multiquark operators does not leave enough room for the occurrence of a possible globally confined structure of the corresponding states, since interactions among hadronic clusters are

\footnotetext{
${ }^{1}$ The cluster reducibility property of multiquark operators was first emphasized in 1980 by Jan Stern, who called attention, through seminars, to that aspect. He did not, however, leave any written article about the subject. The proof presented in this article is based on the arguments developed by Jan Stern.
} 
expected to be nonconfining. Possible implications of this result and connections with other investigations about multiquark states will be discussed in Sec. IV. Sections II and III are devoted to the details of the proof of cluster reducibility in the $S U(3)$ and $S U\left(N_{c}\right)$ cases, respectively. A summary is presented in Sec. V.

\section{MULTIQUARK OPERATORS}

General gauge-invariant multiquark operators are constructed by use of path-ordered gluon-field phase factors, also called Wilson lines, which have the form

$$
U_{b}^{a}\left(C_{y x}\right)=\left(P e^{-i g \int_{C_{y x}} d z^{\mu} T^{B} A_{\mu}^{B}(z)}\right)_{b}^{a},
$$

where $C_{y x}$ is an oriented curve going from $x$ to $y, A^{B}{ }_{\mu}$ are the gluon fields $(B=1, \ldots, 8), T^{B}$ are the generators of the color gauge group $S U(3)$ in the fundamental representation, $g$ is the QCD coupling constant, and $P$ represents the path-ordering operation, meaning that the gluon fields are ordered according to their position on the line $C_{y x}$; the integration runs along the line $C_{y x}$ from $x$ to $y$ [9-12]. The phase factors $U\left(C_{y x}\right)$ are the color parallel transporters of the theory along the lines $C_{y x}$ [13]. Examples of line $C_{y x}$ are shown in Fig. 1; straight lines are generally chosen for their simplicity and also for their adequacy in lattice calculations [14].

For mesonic and baryonic gauge-invariant operators, one has the following constructions,

$$
\begin{gathered}
M=\bar{q}_{a}(y) U^{a}{ }_{b}\left(C_{y x}\right) q^{b}(x), \\
B=\epsilon_{a b c} U_{d}^{a}\left(C_{x y}\right) q^{d}(y) U^{b}{ }_{e}\left(C_{x t}\right) q^{e}(t) U^{c}{ }_{f}\left(C_{x z}\right) q^{f}(z),
\end{gathered}
$$

quark flavor and spin indices being omitted and where $\epsilon$ is the three-dimensional Levi-Civita symbol. A corresponding pictorial representation is given in Fig. 2.

The choice of the line types in representations (2) and (3) is arbitrary, provided they remain continuous with rather smooth variations. Physical quantities should not depend on that choice, which would show up only in the corresponding wave function of states. This can be verified in the case of bound-state energies, which are controlled by the properties of Wilson loops at large time separations [14]. In QCD, Wilson-loop averages are expected to respect
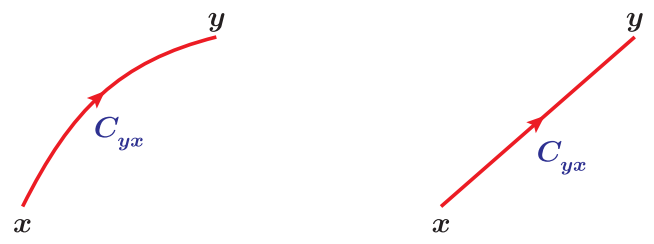

FIG. 1. Examples of line $C_{y x}$.

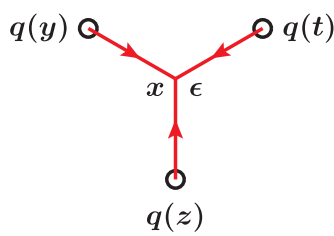

FIG. 2. Pictorial representation of mesonic and baryonic gaugeinvariant operators, with phase factors running along straight lines; in the baryonic case, $\epsilon$ is the Levi-Civita symbol, put here as a reminder of the completely antisymmetric structure of the junction vertex of the three phase-factor lines.

the area law for large rectangular contours and the minimal surface property for more general contours [14-16]. Deformations of the phase-factor lines inside the states are completely projected, when the time interval goes to infinity, onto the wave functions (cf. the end of Appendix A of Ref. [16]).

Similar constructions can be done for the multiquark operators. Pictorial representations of tetraquark, pentaquark, and hexaquark operators are shown in Fig. 3. (For simplicity, the case of hexaquark operators with three quark and three antiquark fields will be omitted in this article; it can be treated in a similar way as the other cases considered here.)

The proof of the cluster reducibility property of multiquark operators is based on the observation that the pathordered phase factors are elements of the color gauge group $S U(3)$. Therefore, they satisfy the group product law

$$
U_{b}^{a}\left(C_{z y}\right) U^{b}{ }_{c}\left(C_{y x}\right)=U^{a}{ }_{c}\left(C_{z y x}\right),
$$

with the determinant of their matrix representation equal to 1 (cf. Appendix C of Ref. [17]):

$$
\begin{aligned}
& \operatorname{det}\left(U\left(C_{y x}\right)\right)=1 \\
& =\frac{1}{3 !} \epsilon_{a_{1} a_{2} a_{3}} \epsilon^{b_{1} b_{2} b_{3}} U^{a_{1}} b_{1}\left(C_{y x}\right) U^{a_{2}}{ }_{b_{2}}\left(C_{y x}\right) U^{a_{3}} b_{3}\left(C_{y x}\right) .
\end{aligned}
$$

A pictorial representation of Eq. (4) is shown in Fig. 4.

We consider here the example of the tetraquark operator [Fig. 3(a)], which has the expression

$$
\begin{aligned}
T= & \bar{q}_{a_{1}}\left(u_{1}\right) U^{a_{1}} c_{1}\left(C_{u_{1} x}\right) \bar{q}_{a_{2}}\left(u_{2}\right) U_{c_{2}}^{a_{2}}\left(C_{u_{2} x}\right) \\
& \times \epsilon^{c_{1} c_{2} c_{3}} U^{d_{3}} c_{c_{3}}\left(C_{y x}\right) \epsilon_{d_{1} d_{2} d_{3}} \\
& \times U^{d_{2}} b_{2}\left(C_{y v_{2}}\right) q^{b_{2}}\left(v_{2}\right) U^{d_{1}} b_{1}\left(C_{y v_{1}}\right) q^{b_{1}}\left(v_{1}\right) .
\end{aligned}
$$

One multiplies $T$ with the determinant (5), corresponding to a phase factor taken along a line $\Gamma_{x y}$, joining $y$ to $x$. The shape of $\Gamma$ is arbitrary, but for simplifying purposes, it could be taken of the same form as the line $C_{y x}$, with opposite orientation. Upon using identities of the type 


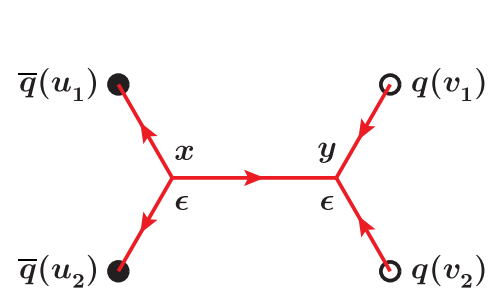

(a)

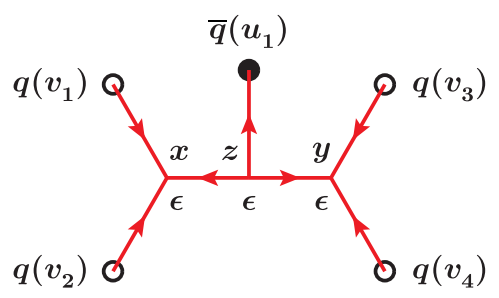

(b)

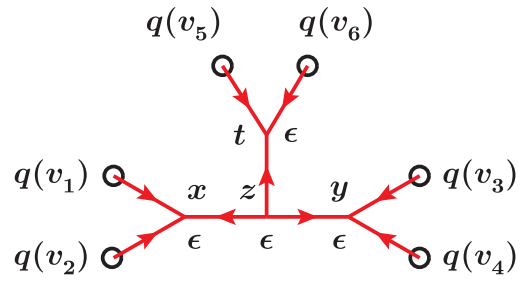

(c)

FIG. 3. Pictorial representation of (a) tetraquark, (b) pentaquark, and (c) hexaquark operators. Hexaquark operators with three quark and three antiquark fields are omitted.

$$
\epsilon^{f_{1} f_{2} f_{3}} \epsilon_{a_{1} a_{2} a_{3}}=\delta_{a_{1}}^{f_{1}} \delta_{a_{2}}^{f_{2}} \delta_{a_{3}}^{f_{3}}+\sum_{k_{i}}(-1)^{p} \delta_{a_{1}}^{f_{k_{1}}} \delta_{a_{2}}^{f_{k_{2}}} \delta_{a_{3}}^{f_{k_{3}}},
$$

where the sum runs over all permutations of the indices $k_{i}$ with $(-1)^{p}$ representing the parity of the permutations, and choosing each time one $\epsilon$ from the $T$ and another from the determinant, one ends up with the expression

$$
\begin{aligned}
T= & \operatorname{tr}\left(U\left(\Gamma_{x y}\right) U\left(C_{y x}\right)\right) \times\left[\bar{q}\left(u_{1}\right) U\left(C_{u_{1} x}\right) U\left(\Gamma_{x y}\right) U\left(C_{y v_{1}}\right) q\left(v_{1}\right)\right] \times\left[\bar{q}\left(u_{2}\right) U\left(C_{u_{2} x}\right) U\left(\Gamma_{x y}\right) U\left(C_{y v_{2}}\right) q\left(v_{2}\right)\right] \\
& -\left[\bar{q}\left(u_{1}\right) U\left(C_{u_{1} x}\right) U\left(\Gamma_{x y}\right) U\left(C_{y x}\right) U\left(\Gamma_{x y}\right) U\left(C_{y v_{1}}\right) q\left(v_{1}\right)\right] \times\left[\bar{q}\left(u_{2}\right) U\left(C_{u_{2} x}\right) U\left(\Gamma_{x y}\right) U\left(C_{y v_{2}}\right) q\left(v_{2}\right)\right] \\
& -\left[\bar{q}\left(u_{1}\right) U\left(C_{u_{1} x}\right) U\left(\Gamma_{x y}\right) U\left(C_{y v_{1}}\right) q\left(v_{1}\right)\right] \times\left[\bar{q}\left(u_{2}\right) U\left(C_{u_{2} x}\right) U\left(\Gamma_{x y}\right) U\left(C_{y x}\right) U\left(\Gamma_{x y}\right) U\left(C_{y v_{2}}\right) q\left(v_{2}\right)\right] \\
& +\operatorname{tr}\left(U\left(\Gamma_{x y}\right) U\left(C_{y x}\right)\right) \times\left[\bar{q}\left(u_{1}\right) U\left(C_{u_{1} x}\right) U\left(\Gamma_{x y}\right) U\left(C_{y v_{2}}\right) q\left(v_{2}\right)\right] \times\left[\bar{q}\left(u_{2}\right) U\left(C_{u_{2} x}\right) U\left(\Gamma_{x y}\right) U\left(C_{y v_{1}}\right) q\left(v_{1}\right)\right] \\
& -\left[\bar{q}\left(u_{1}\right) U\left(C_{u_{1} x}\right) U\left(\Gamma_{x y}\right) U\left(C_{y v_{2}}\right) q\left(v_{2}\right)\right] \times\left[\bar{q}\left(u_{2}\right) U\left(C_{u_{2} x}\right) U\left(\Gamma_{x y}\right) U\left(C_{y x}\right) U\left(\Gamma_{x y}\right) U\left(C_{y v_{1}}\right) q\left(v_{1}\right)\right] \\
& -\left[\bar{q}\left(u_{1}\right) U\left(C_{u_{1} x}\right) U\left(\Gamma_{x y}\right) U\left(C_{y x}\right) U\left(\Gamma_{x y}\right) U\left(C_{y v_{2}}\right) q\left(v_{2}\right)\right] \times\left[\bar{q}\left(u_{2}\right) U\left(C_{u_{2} x}\right) U\left(\Gamma_{x y}\right) U\left(C_{y v_{1}}\right) q\left(v_{1}\right)\right] .
\end{aligned}
$$

The tetraquark operator is thus reexpressed in the form of a combination of six terms, each of which is a product of mesonic clusters. The term with the trace operation, which appears twice, represents a Wilson loop along the closed

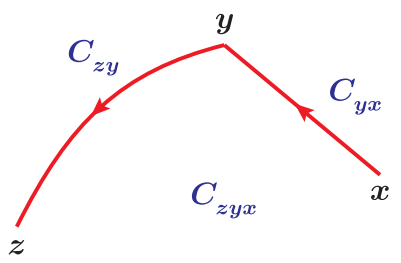

FIG. 4. Group product law of phase factors: the product of the two phase factors along the lines $C_{y x}$ and $C_{z y}$, respectively, is equal to the phase factor along the composite line $C_{z y x}$. contour $\Gamma_{x y} C_{y x}$, which is an independent gauge-invariant operator. In case the line $\Gamma_{x y}$ is chosen of the same shape as $C_{y x}$, using the generally admitted backtracking relation [18]

$$
U_{b}^{a}\left(C_{y x}\right) U_{c}^{b}\left(C_{x y}\right)=\delta_{c}^{a},
$$

the Wilson loop reduces to its normalization constant (which here assumes the value 3). Equation (8) is displayed in pictorial form in Fig. 5, where the line $\Gamma_{x y}$ has been taken of the same shape as the line $C_{y x}$ (a straight line). It is also worthwhile to notice that, if the various internal lines of the mesonic operators are approximated by a common line of the same shape, the decomposition will contain only two terms, corresponding to the two different possibilities of producing the mesonic clusters. 


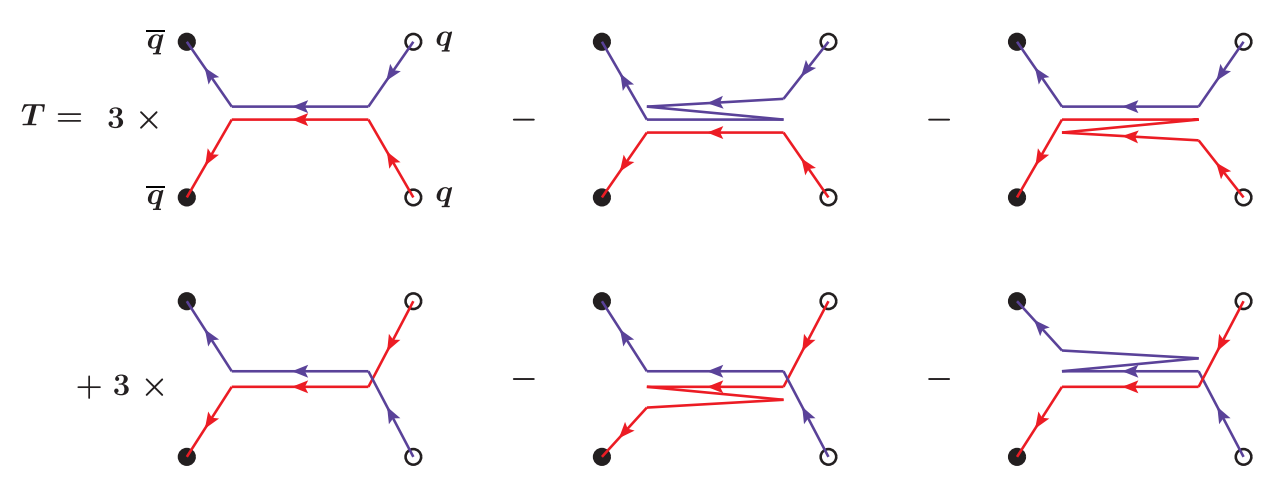

FIG. 5. Decomposition of the tetraquark operator into a combination of products of mesonic operators.

The above procedure can also be applied to the pentaquark and hexaquark operators. For the pentaquark case, one may multiply the operator with the determinant of the phase factor considered along the line $y z$ of Fig. 3(b). One obtains decompositions into products of mesonic and baryonic operators of the type of Fig. 6, with other terms similar to those in Fig. 5.

For the hexaquark case, one may multiply the operator with the determinant of the phase factor considered along the line $y z$ of Fig. 3(c). One obtains decompositions into products of baryonic operators of the type of Fig. 7, with other terms similar to those in Fig. 5.

As a general rule, the procedure of the insertion of the determinant along certain lines leads to cluster reducibility whenever there are two $Y$-shaped junctions linked by a phase-factor line. In this connection, one observes that the usual hybrid operators, which are obtained by insertions inside conventional mesonic and baryonic operators of the gluon field strength $G_{\mu \nu}^{B} T^{B}$, are not cluster reducible, since

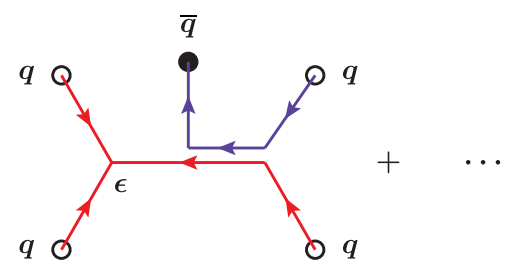

FIG. 6. Decomposition of the pentaquark operator into a combination of products of mesonic and baryonic operators; the ellipsis indicates other types of the link, as in Fig. 5.

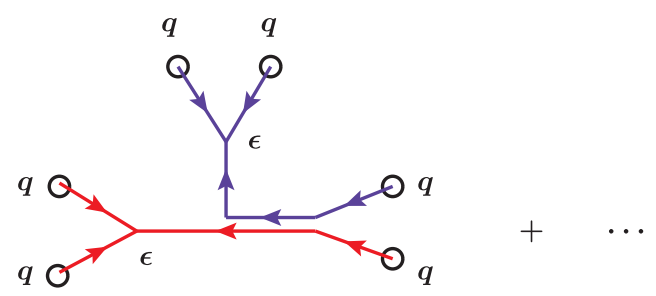

FIG. 7. Decomposition of the hexaquark operator into a combination of products of baryonic operators; the ellipsis designates other types of the link, as in Fig. 5.
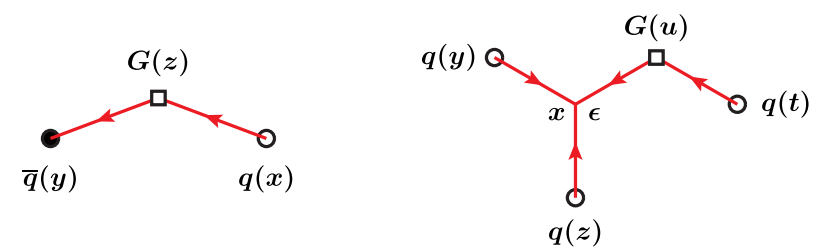

FIG. 8. Mesonic and baryonic hybrid operators; $G$ is the gluon field strength.

they do not contain two $Y$-shaped junctions. Typical hybrid operators are

$M_{h b, \mu \nu}=\bar{q}_{a}(y) U^{a}{ }_{b}\left(C_{y z}\right)\left(G_{\mu \nu}^{B}(z) T^{B}\right)^{b}{ }_{c} U^{c}{ }_{d}\left(C_{z x}\right) q^{d}(x)$,

$\begin{aligned} B_{h b, \mu \nu}= & \epsilon_{a b c} U^{a}{ }_{d}\left(C_{x y}\right) q^{d}(y) U^{b}{ }_{e}\left(C_{x z}\right) q^{e}(z) U^{c}{ }_{f}\left(C_{x u}\right) \\ & \times\left(G_{\mu \nu}^{B}(u) T^{B}\right){ }_{g}^{f} U^{g}{ }_{h}\left(C_{u t}\right) q^{h}(t) .\end{aligned}$

They are pictorially represented in Fig. 8 .

\section{III. $S U\left(N_{c}\right)$}

The color gauge group $S U(3)$ is often extended to the group $S U\left(N_{c}\right)$, where $N_{c}$ is the dimension of the defining fundamental representation and is treated as a free parameter. It turns out that, for large values of $N_{c}$, the properties of the theory become simplified and the theory can be studied through an expansion in powers of the parameter $1 / N_{c}[3,19,20]$; in particular, inelasticity and screening effects become nondominant, and the leading terms display more clearly the confining properties of the theory. It is therefore useful to also have representations of multiquark operators in the $S U\left(N_{c}\right)$ case.

While at the level of ordinary mesonic operators no changes occur, baryonic operators undergo modifications due the necessity of constructing completely colorantisymmetric representations. The Levi-Civita symbol is now replaced by its $N_{c}$-dimensional version, and this requires the junction of $N_{c}$ phase-factor lines. A pictorial representation of the two operators is shown in Fig. 9. 


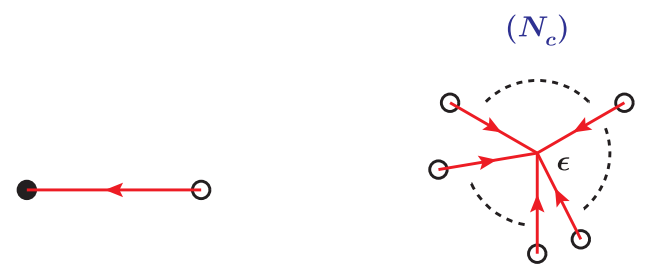

FIG. 9. Mesonic and baryonic operators in the $S U\left(N_{c}\right)$ case; $\epsilon$ is the Levi-Civita symbol in $N_{c}$ dimensions.

For large values of $N_{c}$, mesons become free noninteracting particles, with masses that are independent of $N_{c}$ at leading order [20]. The masses of baryons, due to the increasing number of associated quarks, increase like $N_{c}$; however, the shapes, determined by electric charge density, and sizes of baryons remain almost unchanged [20].

In a similar way, the $Y$-shaped junctions of the $S U(3)$ case are replaced by junctions of $N_{c}$ phase-factor lines. This feature leads, however, to multiple choices for the construction of multiquark operators. Considering, for instance, the tetraquark case, Fig. 3(a), one may replace the two external quarks and their accompanying lines by $\left(N_{c}-1\right)$ quarks and lines and similarly for the antiquarks, the two junction points being linked together by a single phase-factor line. However, one may also choose $2\left(N_{c}-2\right)$ external quarks and antiquarks, with their junction points being now linked together by two phase-factor lines, and so forth. The extreme case corresponds to a representation where one has two pairs of external quarks and antiquarks, with junction points linked together by $\left(N_{c}-2\right)$ phase-factor lines. Thus, one obtains a sequence of tetraquark-generalizing $\left[2\left(N_{c}-1\right)\right]$-quark,
$\left[2\left(N_{c}-2\right)\right]$-quark, $\ldots$, and $\left[2\left(N_{c}-\left(N_{c}-2\right)\right)=4\right]$-quark operators. Pictorial representations of the two extremal cases are shown in Fig. 10.

Each of the operators obtained above corresponds to a different state. As is the case with ordinary baryons, it is expected that states with increasing numbers of quarks and antiquarks will have increasing values of masses with $N_{c}$. The state corresponding to the extreme case with two quarks and two antiquarks would have the smallest mass among the many possibilities that are encountered.

The pentaquark operator, like in the tetraquark case, also has multiple extensions, as shown in Fig. 11. In one extreme case, one has $2\left(N_{c}-1\right)$ external quarks and $\left(N_{c}-2\right)$ external antiquarks, while in the other extreme case, one has $\left(N_{c}-1\right)+2$ quarks and one antiquark.

Hexaquark operators are constructed for general $N_{c}(\geq 3)$ as in the previous cases. Their pictorial representations are shown in Fig. 12.

Properties of possibly existing tetraquarks have been studied for large values of $N_{c}$ in Refs. [21-29]. General cases of multiquarks have been considered in Ref. [30].

The cluster reducibility property of multiquark operators shown in the $S U(3)$ case can also be extended to the general $S U\left(N_{c}\right)$ case. The main ingredients of the proof, Eqs. (4), (5), and (7), are naturally extended to that case [17]. For tetraquarks, the first operator in Fig. 10 decomposes into a combination of products of $\left(N_{c}-1\right)$ mesonic operators; the last operator of that figure decomposes into a combination of products of two mesonic operators and eventually of Wilson loops, according to the choices of the types of the internal phase-factor lines. Similar decompositions occur for the intermediate cases of Fig. 10.
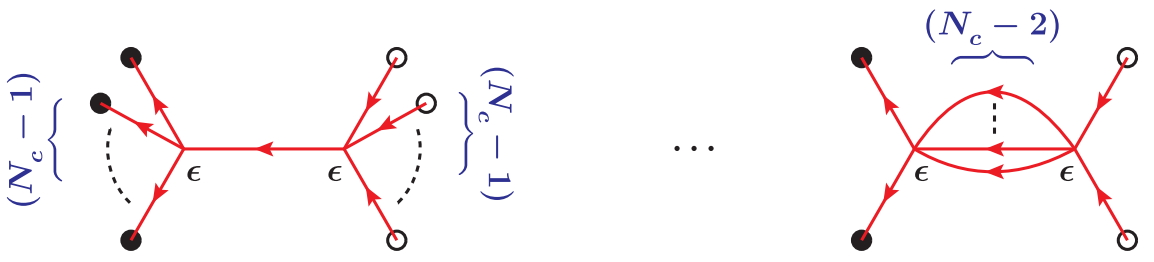

FIG. 10. Tetraquark operators in the $S U\left(N_{c}\right)$ case, where two extreme cases are shown. The first diagram contains $\left(N_{c}-1\right)$ quarks and $\left(N_{c}-1\right)$ antiquarks, with a single link between the two subsystems. The last diagram contains two quarks and two antiquarks, with $\left(N_{c}-2\right)$ links between the subsystems.

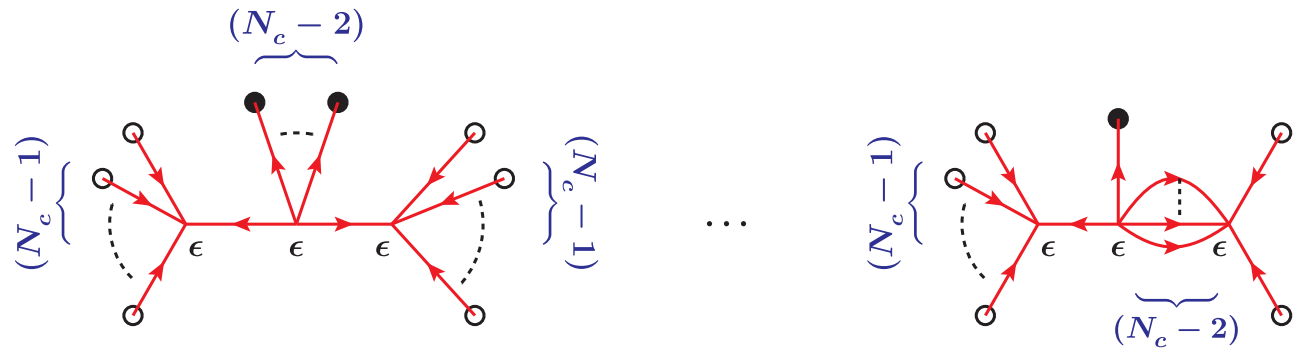

FIG. 11. Pentaquark operators in the $S U\left(N_{c}\right)$ case, with two extreme cases shown. The first diagram contains $2\left(N_{c}-1\right)$ quarks and $\left(N_{c}-2\right)$ antiquarks. The last diagram contains $\left(N_{c}-1\right)+2$ quarks and one antiquark. 

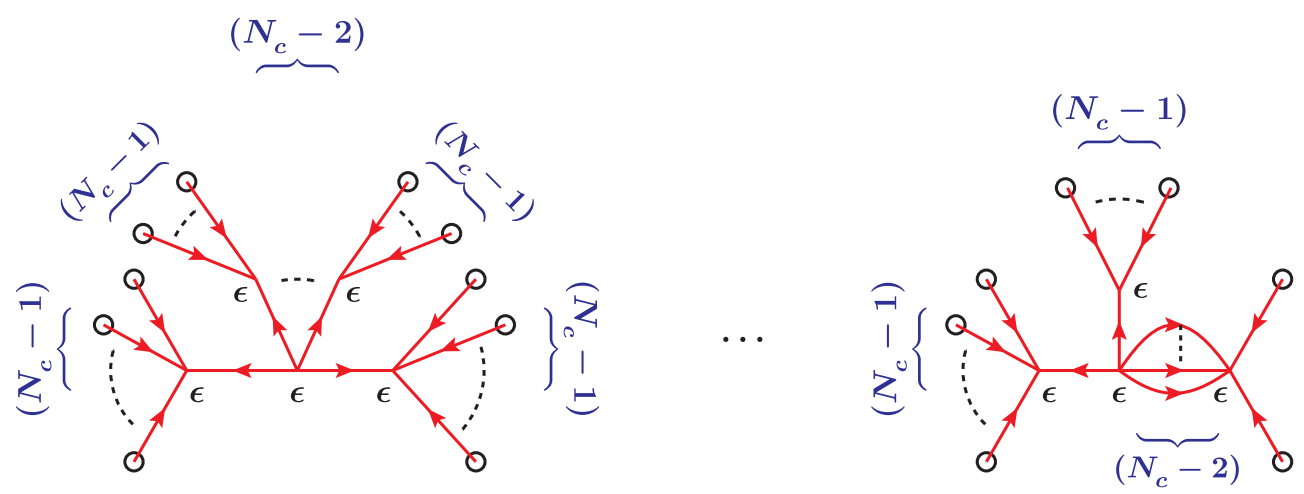

FIG. 12. Hexaquark operators in the $S U\left(N_{c}\right)$ case, with two extreme cases shown. The first diagram contains $N_{c}\left(N_{c}-1\right)$ quarks; at the central junction point, apart from the bifurcation into the two horizontal lines, there are bifurcations into $\left(N_{c}-2\right)$ lines, which in turn bifurcate each into $\left(N_{c}-1\right)$ lines. The last diagram contains $2\left(N_{c}-1\right)+2$ quarks.

For pentaquarks, the first operator in Fig. 11 decomposes into a combination of products of $\left(N_{c}-2\right)$ mesonic operators and one baryonic operator. The last operator of that figure decomposes into a combination of products of one mesonic operator and one baryonic operator and eventually of Wilson loops.

In the case of hexaquarks, the first operator in Fig. 12 decomposes into a combination of products of $\left(N_{c}-1\right)$ baryonic operators. The last operator of that figure decomposes into a combination of products of two baryonic operators and eventually of Wilson loops.

\section{DISCUSSION}

Since hadronic clusters do not mutually have confiningtype interactions, one would be tempted to conclude that the global effective interactions that govern the emergence of multiquark bound states or resonances should be of the molecular type [31-36]. However, this issue requires a more refined analysis.

Molecular-type descriptions of exotic states are generally based on effective field theories, using hadronic degrees of freedom. These theories are essentially low-energy theories, which become less convergent at short distances, necessitating the inclusion of higher-order contributions with an increasing number of low-energy constants [35-39]. The multiquark scheme uses, in essence, quark degrees of freedom and therefore is more adapted to describe short-distance regimes of hadronic clusters. The main practical question that emerges is therefore that of the determination of the domains of dominance, inside an exotic state, of each of the preceding pictures.

In order to study the internal dynamics that are at work inside exotic states, calculations have been undertaken in the past in lattice theory by displaying the gauge-field configurations that are dominant in correlation functions of multiquark operators [40-44]. It turns out that, when the distance between two hadronic clusters is smaller than the mean size of each hadron, it is the connected $Y$-shaped-type configuration that dominates, while in the opposite case, it is the two disconnected hadronic cluster-type configurations that are dominant. The static interquark confining potential is then determined by the minimal value of the total lengths of the strings of each case. These results confirm the fact that the multiquark scheme, based essentially on the string-junction or diquark picture, even though globally nonconfining because of the presence or emergence of hadronic clusters, remains a basic ingredient for a precise description of the multiquark state.

The above results have led to the adoption of similar potential models in nonrelativistic and semirelativistic approaches, based on the idea of the partitioning of configuration space, according to the dominance region of each type of potential [45-56]. One of the advantages of these models is the absence of unphysical long-range van der Waals forces, which unavoidably occur in additive quark models with confining potentials.

The concept of partitioning the configuration space, at least in a schematic sense, according to the dominance of the various geometrical configurations of the multiquark systems, appears as providing the most optimal framework which is compatible with the general cluster reducibility property of multiquark operators.

One therefore naturally arrives at the following picture of exotic states. The latter should be described by two complementary schemes, each valid in a separate region of configuration space: the multiquark or diquark scheme, valid in regions where the hadronic clusters are close to each other, and the molecular-type scheme, valid in regions where the hadronic clusters are well separated from each other; a crossover should prevail at the frontier region; the weight of each configuration would depend on the quark masses and flavors, as well as on the sectors of quantum numbers.

According to the weight of each scheme, approximate descriptions might be considered at a starting stage, in particular, when one of the schemes is overdominant. In case of comparable weights, mixtures of the two schemes offer other possibilities [57]. 
Recent review articles on exotic states can be found in Refs. [36,58-63].

\section{SUMMARY}

The cluster reducibility property of multiquark operators provides a general proof of the nonexistence of completely confined or compact multiquark states. Rather than eliminating the multiquark scheme from the description of multiquark states, taking into account analyses based on lattice and numerical calculations, it streamlines the role played by the various participating operators, according to a qualitative partitioning of configuration space. Existing multiquark states, whether bound states or resonances, would be schematically composed of two layers: an inner core, having a structure governed by a connected string-junction-type interaction, and an outer shell, having a hadronic molecular-type structure. The weight of each layer depends on the masses and flavors of the quarks and on the sectors of quantum numbers that are considered. This unified scheme might provide a better understanding of the structure of multiquark states.

\section{ACKNOWLEDGMENTS}

We thank Hans Günter Dosch for enlightening discussions. D. M. acknowledges support from the Austrian Science Fund (FWF), Grant No. P29028. D. M. and H. S. are grateful for support under joint CNRS/RFBR Grant No. PRC Russia/19-52-15022. The figures were drawn with the aid of the package Axodraw2 [64].
[1] R. L. Jaffe, Phys. Rev. D 15, 281 (1977).

[2] R. L. Jaffe, Nucl. Phys. A804, 25 (2008).

[3] S. Coleman, Aspects of Symmetry (Cambridge University Press, Cambridge, England, 1985), Chap. 8.

[4] G. C. Rossi and G. Veneziano, Nucl. Phys. B123, 507 (1977).

[5] G. C. Rossi and G. Veneziano, J. High Energy Phys. 06 (2016) 041.

[6] R. Jaffe and F. Wilczek, Phys. Rev. Lett. 91, 232003 (2003).

[7] E. Shuryak and I. Zahed, Phys. Lett. B 589, 21 (2004).

[8] L. Maiani, F. Piccinini, A. D. Polosa, and V. Riquer, Phys. Rev. D 71, 014028 (2005).

[9] S. Mandelstam, Ann. Phys. (N.Y.) 19, 1 (1962).

[10] I. Bialynicki-Birula, Bull. Acad. Pol. Sci., Sér. Sci. Math., Astr. et Phys. 11, 135 (1963).

[11] S. Mandelstam, Phys. Rev. 175, 1580 (1968).

[12] Y. Nambu, Phys. Lett. 80B, 372 (1979).

[13] E. Corrigan and B. Hasslacher, Phys. Lett. 81B, 181 (1979).

[14] K. G. Wilson, Phys. Rev. D 10, 2445 (1974).

[15] Yu. M. Makeenko and A. A. Migdal, Phys. Lett. 97B, 253 (1980).

[16] F. Jugeau and H. Sazdjian, Nucl. Phys. B670, 221 (2003).

[17] A. A. Migdal, Phys. Rep. 102, 199 (1983).

[18] Y. Makeenko, arXiv:hep-th/0001047.

[19] G. 't Hooft, Nucl. Phys. B72, 461 (1974).

[20] E. Witten, Nucl. Phys. B160, 57 (1979).

[21] S. Weinberg, Phys. Rev. Lett. 110, 261601 (2013).

[22] M. Knecht and S. Peris, Phys. Rev. D 88, 036016 (2013).

[23] F.-K. Guo, L. Liu, U.-G. Meißner, and P. Wang, Phys. Rev. D 88, 074506 (2013).

[24] T. D. Cohen and R. F. Lebed, Phys. Rev. D 90, 016001 (2014).

[25] L. Maiani, A. D. Polosa, and V. Riquer, J. High Energy Phys. 06 (2016) 160.

[26] W. Lucha, D. Melikhov, and H. Sazdjian, Phys. Rev. D 96, 014022 (2017).

[27] W. Lucha, D. Melikhov, and H. Sazdjian, Eur. Phys. J. C 77, 866 (2017).
[28] L. Maiani, A. D. Polosa, and V. Riquer, Phys. Rev. D 98, 054023 (2018).

[29] W. Lucha, D. Melikhov, and H. Sazdjian, Phys. Rev. D 98 , 094011 (2018).

[30] L. Maiani, V. Riquer, and W. Wang, Eur. Phys. J. C 78, 1011 (2018).

[31] S. Weinberg, Phys. Rev. 137, B672 (1965).

[32] M. B. Voloshin and L. B. Okun, Pis'ma Zh. Eksp. Teor. Fiz. 23, 369 (1976) [JETP Lett. 23, 333 (1976)].

[33] A. De Rújula, H. Georgi, and S. L. Glashow, Phys. Rev. Lett. 38, 317 (1977).

[34] C. Amsler and N. A. Törnqvist, Phys. Rep. 389, 61 (2004).

[35] M. Pavón Valderrama, Phys. Rev. D 85, 114037 (2012).

[36] F.-K. Guo, C. Hanhart, U.-G. Meißner, Q. Wang, Q. Zhao, and B.-S. Zou, Rev. Mod. Phys. 90, 015004 (2018).

[37] S. Weinberg, Physica (Amsterdam) 96A, 327 (1979).

[38] J. Gasser and H. Leutwyler, Ann. Phys. (N.Y.) 158, 142 (1984).

[39] A. V. Manohar, Lect. Notes Phys. 479, 311 (1977).

[40] H. G. Dosch, Phys. Rev. D 28, 412 (1983).

[41] C. Alexandrou and G. Koutsou, Phys. Rev. D 71, 014504 (2005).

[42] F. Okiharu, H. Suganuma, and T. T. Takahashi, Phys. Rev. Lett. 94, 192001 (2005).

[43] F. Okiharu, H. Suganuma, and T. T. Takahashi, Phys. Rev. D 72, 014505 (2005).

[44] H. Suganuma, T. Iritani, F. Okiharu, T. T. Takahashi, and A. Yamamoto, AIP Conf. Proc. 1388, 195 (2011).

[45] F. Lenz, J. T. Londergan, E. J. Moniz, R. Rosenfelder, M. Stingl, and K. Yazaki, Ann. Phys. (N.Y.) 170, 65 (1986).

[46] J. Carlson and V. R. Pandharipande, Phys. Rev. D 43, 1652 (1991).

[47] G. Martens, C. Greiner, S. Leupold, and U. Mosel, Phys. Rev. D 73, 096004 (2006). 
[48] J. Vijande, A. Valcarce, and J.-M. Richard, Phys. Rev. D 76, 114013 (2007).

[49] J. Vijande, A. Valcarce, and J.-M. Richard, Phys. Rev. D 85, 014019 (2012).

[50] A. V. Manohar and M. B. Wise, Nucl. Phys. B399, 17 (1993).

[51] P. Bicudo, K. Cichy, A. Peters, and M. Wagner, Phys. Rev. D 93, 034501 (2016).

[52] P. Bicudo, J. Scheunert, and M. Wagner, Phys. Rev. D 95, 034502 (2017).

[53] P. Bicudo, M. Cardoso, O. Oliveira, and P. J. Silva, Phys. Rev. D 96, 074508 (2017).

[54] M. Karliner and J. L. Rosner, Phys. Rev. Lett. 119, 202001 (2017).

[55] E. J. Eichten and C. Quigg, Phys. Rev. Lett. 119, 202002 (2017).

[56] C. Quigg, arXiv:1804.04929.

[57] A. Esposito, A. Pilloni, and A. D. Polosa, Phys. Lett. B 758, 292 (2016).
[58] A. Esposito, A. Pilloni, and A. D. Polosa, Phys. Rep. 668, 1 (2017).

[59] R. F. Lebed, R. E. Mitchell, and E. S. Swanson, Prog. Part. Nucl. Phys. 93, 143 (2017).

[60] A. Ali, J. S. Lange, and S. Stone, Prog. Part. Nucl. Phys. 97, 123 (2017).

[61] S. L. Olsen, T. Skwarnicki, and D. Zieminska, Rev. Mod. Phys. 90, 015003 (2018).

[62] M. Karliner, J. L. Rosner, and T. Skwarnicki, Annu. Rev. Nucl. Part. Sci. 68, 17 (2018).

[63] N. Brambilla, S. Eidelman, C. Hanhart, A. Nefediev, C.-P. Shen, C. E. Thomas, A. Vairo, and C.-Z. Yuan, arXiv: 1907.07583.

[64] J. C. Collins and J. A. M. Vermaseren, arXiv:1606.01177.

Correction: The source listing for Ref. [39] was incorrectly presented and has been fixed. 